\title{
Implementing a peer-learning approach for the clinical education of respiratory therapy students
}

\author{
Stephanie Dorner, RRT, CHT, BA ${ }^{1}$, Tara Fowler, RRT, CHT, MHS ${ }^{1}$, Martha Montano, RRT, BSc ${ }^{1}$, \\ Ray Janisse, RRT, $\mathrm{CHT}^{1}$, Mandy Lowe, BScOT, MSc ${ }^{2,3}$, Paula Rowland, OT, $\mathrm{PhD}^{2,4}$
}

\begin{abstract}
S Dorner, T Fowler, M Montano, R Janisse, M Lowe, P Rowland. Implementing a peer-learning approach for the clinical education of respiratory therapy students. Can J Respir Ther 2019;55:21-27. doi: 10.29390/cjrt-2018-022.

Introduction: With recent clinical placement demands exceeding supply, the University Health Network (UHN) Respiratory Therapy (RT) department implemented a 2:1 student-to-preceptor model where a focus on peer learning (PL) becomes a key component of program success. PL can be defined as students learning from and with each other in both formal and informal ways. The shift towards facilitative student-directed models in other health care professions can be seen globally with the literature suggesting that 2:1 models not only support increases in student capacity but also improve the student learning experience through PL strategies. The aim of this study was to explore the perceptions of RT preceptors and students regarding the 2:1 model as an educational strategy in the context of their clinical experience. The study further explored experiences of PL to understand how learning is enabled in RT practice-based education, particularly within 2:1 models.

Methods: A qualitative descriptive study using single-episode semi-structured interviews with RT preceptors $(n=10)$ and students $(n=10)$ was conducted during the 2015-2016 RT student clinical year. Twelve open-ended interview questions were designed to draw out study participants' PL experiences and exploration of issues using a 2:1 model in the context of their clinical experience. Data were recorded, transcribed verbatim, and analyzed using thematic analysis.

Results: The content analysis resulted in two broad themes with respect to the RT 2:1 educational model: "enablers" and "barriers" to a PL approach. The 2:1 model was preferred by students and preceptors early on in the clinical training due to the benefits of PL, whereas opportunities to showcase independent practice was preferred towards the end of their clinical year. Furthermore, careful planning, resources, and supports need to be implemented to augment benefits and diminish potential disadvantages of using a 2:1 model structure.

Conclusion: Participants felt that a 2:1 model strongly contributes to a supportive learning environment and can have a positive influence on the RT student clinical experience at UHN. Along with the improved critical thinking and student engagement opportunities that a 2:1 model offers, increased placement numbers are also supported.
\end{abstract}

Key Words: collaborative learning; 2:1 model; planned peer learning; unplanned peer learning

\section{INTRODUCTION}

Pressures caused by decreases in both hospital and education program funding, along with increases in patient acuity, workload, and student placement demands, have driven institutions to explore different models of clinical education to support their student training [1]. An emerging focus for clinical education programs is one that prepares students for working as a member of an integrated team through immersing them in a model where learning from one another is expected and having an awareness of others perspectives is habitual. One such model features peer learning (PL) as an educational strategy-supported by pairing students from the same year with one clinical preceptor (2:1 model). Professions such as nursing, physiotherapy, occupational therapy, and speech language pathology have already explored the use of 2:1 models that have successfully demonstrated a means of increasing the number of student placements while concurrently supporting the engagement of students in PL [2-7].
Boud et al. [8] highlighted that PL is not a single educational strategy but is instead a pedagogy based on the ideology that learning is active and is inherently a social phenomenon: "Peer learning should be mutually beneficial and involve the sharing of knowledge, ideas and experience between the participants" (p. 3). In pairing students together for clinical training, students have the opportunity to reciprocally learn through facilitated and unfacilitated discussions, collaboration, and interprofessional practice under the guidance of their supervising preceptor. The benefits of PL reported in the literature include an increased depth of knowledge gained from peer discussions, reduced student anxiety, autonomy, and increased self-confidence [4, 5, 9-12]. In addition, peer learners reported benefits of PL interactions to include the exploring of ideas without preceptor interference, a review of learned material, improved communication skills, as well as increased comfort with giving and receiving feedback [2, 9, 13]. However, challenges of a $2: 1$ model have also been thoroughly

${ }^{1}$ Department of Respiratory Therapy, Allied Health, Toronto General Hospital, Toronto, Ontario Canada

${ }^{2}$ Centre for Interprofessional Education, University of Toronto/University Health Network, Toronto, Ontario, Canada; and Department of Occupational

Science and Occupational Therapy, University of Toronto, Toronto, Ontario, Canada

${ }^{3}$ Michener Institute of Education at University Health Network, University Health Network, Toronto, Ontario, Canada

${ }^{4}$ Wilson Centre for Research in Education, University of Toronto/University Health Network, Toronto, Ontario, Canada

Correspondence: Stephanie Dorner, Toronto General Hospital, 200 Elizabeth Street, Toronto, ON M5G 2C4, Canada. E-mail: Stephanie.dorner@uhn.ca

Published online at https://www.cjrt.ca on 15 February 2019

OPEN ACCESS

This open-access article is distributed under the terms of the Creative Commons Attribution Non-Commercial License (CC BY-NC) (http:// creativecommons.org/licenses/by-nc/4.0/), which permits reuse, distribution and reproduction of the article, provided that the original work is properly cited and the reuse is restricted to noncommercial purposes. For commercial reuse, contact editor@csrt.com 
described in the literature. These include additional paperwork for preceptor evaluations, space allocation for more students, interpersonal challenges between students, student-to-student competition for clinical tasks, peer comparison by preceptors, and less opportunity for independent practice $[4,5,9-11]$. Further, while pairing students together might provide the opportunity for students to learn from one another, it does not guarantee that such learning will occur. Attention must be paid to both the structure of the model and the process of learning that the 2:1 model makes possible.

In Ontario, the Respriatory Therapy (RT) field has experienced growth in its accredited programs and opportunities for continuing education [14]. This has led to increases in classroom size and students' competition for clinical placements. To the best of our knowledge, PL has not been explored for RT students in Canada, but if the reported benefits seen in the literature for other health care professions can be replicated for RT clinical education, PL becomes an attractive clinical education tool to help support higher volumes of student clinical placements.

In 2014, the University Health Network (UHN) RT Department collaborated with the Michener Institute of Applied Health Sciences to study innovative clinical education models that would facilitate an increased number of student placements. A PL educational strategy was selected using a 2:1 model-two same-year students paired with one clinical preceptor for the 2015-2016 RT student clinical year. While there is much to be appreciated about PL within other professions, there is value in a profession-specific exploration of the 2:1 model and the phenomenon of PL given the nature of RT clinical work can be unpredictable and is often dictated by time pressure and constraints. Furthermore, the mediators, mechanisms, and processes involved in running an effective 2:1 model are not clearly defined in the existing literature, thus leaving much uncertainty if a similar model could be as beneficial across different professions. The aim of this study was to describe our 2:1 model and explore RT participants' experiences of PL as an educational strategy to understand how to further enable it in RT clinical education.

\section{DESCRIPTION OF THE UHN RT CLINICAL EDUCATION MODEL}

The Model

A 2:1 model (two same-year students working collaboratively under the supervision of a single preceptor) was introduced for the 2015-2016 RT student clinical year. Sixteen students with placements at UHN were informed of their peer pairing for clinical placement toward the end of their didactic year. Michener faculty determined the peer pairings based on personality and academic performance. Informing students of their peer partner prior to coming to UHN for their clinical training gave peers the opportunity to practice working together during their summer simulation semester.

RT students completed 21 of 31 weeks of clinical exposure directly at UHN. This study was conducted in the third and final year of the RT program during their clinical placement experience. UHN is a large urban network made up of three acute care hospitals, one rehabilitation program over five sites, and a learning institute. The majority of student clinical exposure is supported by the acute care hospitals developing clinical skills on the wards units, intensive care units (ICU), operating rooms (OR) and pulmonary function testing laboratory (PFT lab). Table 1 provides an overview of UHN, and Figure 1 shows the RT clinical year at a glance.
Peer pairs were given the same schedule throughout the entire clinical year. For their ICU ward rotations, students were paired with a single preceptor in a 2:1 model. Each pair of students was supervised by an RT staff designated as a preceptor and students shared in the learning opportunities at the beginning of the clinical experience. Compared with the traditional 1:1 student-to-preceptor model (1:1 model) in which preceptors directly teach the students, preceptors were to facilitate conversations between the students in relation to patient care and support student collaboration. With time, comfort and skill development students transitioned to working independently. Thus, based on participant feedback the clinical model transitioned organically to a 1:1 model towards the end of the clinical year, but because student pairs remained scheduled in the same clinical area, PL strategies persisted. In two instances where workload and space constraints were a concern, such as in the pulmonary function lab and OR clinical areas, a 1:1 model was used. However, opportunities for unplanned PL still occurred through informal discussions during downtimes and breaks as a result of their coinciding schedules. Table 2 lists the commonly described RT practices by both students and preceptors that were performed utilizing a PL structure.

\section{FIGURE 1}

Number of weeks of clinical placement by practice area.

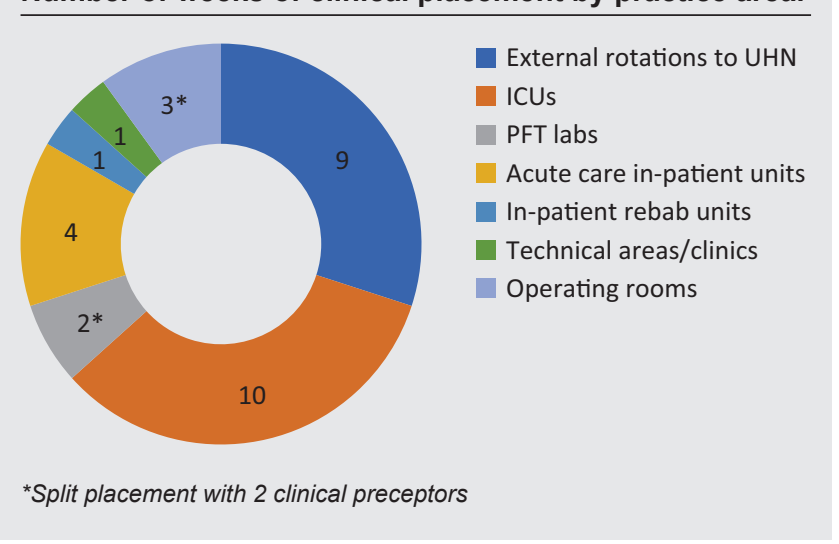

\section{TABLE 2}

\section{Commonly identified peer-learning tasks}

\section{Chart reviews}

Documentation

End of shift reports

Bronchoscopy set up and assist

Ventilator rounds

Arterial blood gas interpretations

Patient care management

Tracheostomy changes and care

Intubation set-up and assist

Respiratory assessment

Equipment assembly/troubleshooting

Debriefing patient care

TABLE 1

Overview of UHN sites supporting the 2015-2016 UHN RT students for clinical training

\begin{tabular}{|c|c|c|c|c|c|}
\hline UHN Site & $\begin{array}{l}\text { Acute care } \\
\text { beds }\end{array}$ & $\begin{array}{l}\text { Critical } \\
\text { care beds }\end{array}$ & $\begin{array}{l}\text { Operating } \\
\text { rooms }\end{array}$ & $\begin{array}{l}\text { Long-term rehabilitation or } \\
\text { complex continuing care beds }\end{array}$ & Clinics \\
\hline Toronto General Hospital & 433 & 59 & 20 & N/A & PFT \\
\hline Toronto Western Hospital & 281 & 22 & 13 & N/A & PFT, Respirology, Asthma and Airway Center \\
\hline Princess Margaret Hospital & 129 & N/A & 2 & $\mathrm{~N} / \mathrm{A}$ & $\mathrm{N} / \mathrm{A}$ \\
\hline Toronto Rehab - Bickle Centre & $\mathrm{N} / \mathrm{A}$ & N/A & $\mathrm{N} / \mathrm{A}$ & 208 & N/A \\
\hline
\end{tabular}


The educational strategy: introducing and reinforcing PL as part of the $2: 1$ model

UHN's PL approach was formally introduced to students during their 5-day hospital orientation. Students were introduced to the theory of PL and PL exercises were integrated into orientation activities. Peer pairs defined, and documented on posters, the ground rules to achieve success in their working pair. These ground rules included strategies about how they would communicate, collaborate, and allocate tasks. This activity also provided an opportunity to discuss their concerns and anxieties about working in a 2:1 model. As a larger group, the students developed a poster for preceptors highlighting expectations on how they would like to be supported; all posters were displayed in the RT department.

Staff were introduced to the 2:1 model and PL strategies during an annual professional development day. The current literature on the benefits and challenges that the model posed were discussed. In addition, a voluntary PL education day was also offered to staff to provide further education about effective facilitation, communication, and feedback skills. Role playing scenarios were performed to explore how a preceptor's role changes with a PL approach-this set the stage for discussions around the unique factors that the health care setting may present.

Four types of PL methods supported students throughout their clinical semester. These included protected preceptor days, planned PL activities (PPL), facilitated PL, and unplanned PL (see Table 3 for PL method descriptions).

\section{METHODS}

\section{Participants and design}

Research ethics board (REB) approval was obtained from UHN on 22 December 2015 and from the Michener Institute of Applied Health Sciences on 15 January 2016. A qualitative descriptive study using a short demographic questionnaire and single episode semi-structured interviews with preceptors $(n=10)$ and students $(n=10)$ was conducted during the 2015-2016 RT student clinical year. The interview questions were designed to explore how a 2:1 model can facilitate PL in the RT clinical environment. Informed consent was required to participate in this study. Study participants were Michener Institute RT students assigned to UHN as their primary site for clinical education and UHN RT staff. RT staff who participated had at a minimum acted in a preceptor role for greater than two consecutive days with the same student pair.

\section{TABLE 3}

\section{Types of peer learning used in the RT clinical model}

Protected Preceptor not having a designated clinical caseload work preceptor day with students to focus on orientation, development, and education to a specific area. Preceptors and students work through structured activities and have limited clinical workload throughout the shift. One to two protected preceptor days were allocated for each pair during the start of each clinical area.

Planned Half-day activities facilitated by the student coordinator for peer-learning all 16 students. These are used to review important concepts activities or competencies with limited exposures. Students are sent topics 6-8 weeks in advance and are divided into peer groups to develop the preparation material; groups often did not include their peer pairing to expand on opportunities for learning from other peers. Students prepare the material, lead, and deliver the education to other students. New knowledge is then applied to problem based case studies. Facilitated Learning activities that occur at the bedside with the preceptor peer learning and students. Preceptors take a role in facilitating discussions, debriefing, or skill development.

Unplanned Learning occurring by two or more peers talking, observing, peer learning and simply working with each other.

\section{Procedure}

Information regarding the study was circulated via email and study flyers. To ensure no undue pressure to participate, or perception of coercion, qualifying participants were asked to contact the RT administrator. The anonymity of interested participants was maintained from the RT leadership team.

Interested participants were approached in February and March 2016, three-quarters of the way through the 2015-2016 clinical year. This ensured they had experience with the 2:1 student-to-preceptor model, PL strategies, and PPL activities. Pilot interviews were undertaken to ensure questions conveyed an open atmosphere where participants could express feelings without constraint [15]. The interviewer was an RT staff member from UHN and had affiliation with the study. However, for the purposes of this study, the interviewer had been excluded from acting as a staff preceptor during the clinical year and had no previous association with the clinical experiences of the students. To mitigate any potential power imbalances between the interviewer and the participants, the interviewer was not involved in the hiring of staff and students and had no power relationship with RTs participating in the study.

After securing informed consent from the participant, one-on-one, face-to-face semi structured interviews lasting up to an hour were held in a private room and audio recorded, along with field notes completed by the interviewer. For consistency, the same interviewer conducted all 20 interviews. Semi-structured questions were utilized to draw out study participants' descriptions and perspectives on PL in the context of their clinical experience. Data were stored in accordance with UHN REB rules and regulations.

\section{Data analysis}

The audio-recorded data were transcribed verbatim into written form for analysis by a third-party transcriptionist. Data of the open-ended questions were manually coded using thematic analysis to identify implicit and explicit ideas within the data. Ideas dealing with the same content generated the initial categories and then were reduced into broader categories taking into account the research question. This approach was used because the researchers were aiming to explore commonalities, differences, and the emergence of new ideas and themes.

\section{RESULTS}

\section{Defining success of an RT clinical educational model}

A clear distinction emerged between student and preceptor responses when asked to define clinical educational success. Throughout their clinical year students are required to attain proficiency in all elements of a clinical competency set out by the National Competency Framework. Clinical competencies are predetermined tasks/skills in which students must demonstrate their knowledge, understanding, and performance of that task/skill to an expected degree of proficiency. Successful achievement of these competencies by students is required to challenge the exam needed for credentialing/licensing. Despite the responsibility falling on the preceptors for evaluating students on each competency as outlined by the evaluation criteria, their defined success of a clinical education program had a "qualitative" emphasis. The responses from preceptors focused on students' ability to apply critical thinking to their patient management, effective communication, team collaboration, and understanding of how respiratory care may affect the patient's other organ systems-the bigger picture. The students' definition of success had a more "quantitative" composition and focused on the acquisition of skills, completion of required competencies, and being able to independently manage the tasks of a patient caseload.

Preceptor 7: "It's understanding why you are doing what you're doing. If you can't put the global picture together, then you're not ready.... thinking critically, working confidently, and safely."

Student 8: "...working independently, gaining confidence, and successfully getting all of your competencies signed off." 
This suggests some potential tension in the PL approach, particularly around issues of assessment of students' competencies. While the 2:1 model encourages learning between peers, eventual decisions about competency are made on an individual basis.

\section{Exploring barriers and enablers to PL}

Content analysis of the interviews resulted in two broad themes with respect to the 2:1 model in the RT program: "enablers" and "barriers". Table 4 lists the main categories within these two themes.

\section{Theme 1: Enablers}

The main categories of reported "enablers" included supportive learning environment and the clinical education model design.

\section{Supportive learning environment}

The most stated enabler by study participants was the supportive learning environment that it fostered. Students cited reduced anxiety starting off their clinical year by having a peer with whom to enter into hospital-based learning ("starting clinical"). They appreciated using one another as a support system and for sharing knowledge and ideas. The opportunity to discuss patient care management, ask one another questions, and reflect on their answers gave them the confidence to articulate patient care plans. They were able to explore questions that they may have been too embarrassed to ask for fear of being perceived as "silly or stupid" in front of their preceptor. Furthermore, viewing fellow peer achievements encouraged students to continue the development of their skills.

Student 14: "I feel like even before starting clinical, I remember talking to my other classmates who went to different sites and who were going to be by themselves; they were freaking out like 'I can't believe clinical is tomorrow' but then I felt calm because I was going to be with my partner so we would have each other as support."

Student 9: "You get to talk through things a lot more comfortably and then you build on each other's ideas. So if I wasn't considering something, my partner would chime in, or the other way around... We definitely were able to learn from each other."

Preceptor 10: "I think when you have a buddy who you're comfortable with, you're much more relaxed and you're more able to absorb the information that is being thrown at you."

The clinical education model design

Students received a formal introduction to PL during their orientation week for clinical placement. During this time, the benefits and challenges of the 2:1 model were discussed and students were given the opportunity to confer on how they would deal with task allocation,

\section{TABLE 4}

Enablers and barriers to successful implementation of a 2:1 model in the RT clinical setting

\begin{tabular}{|c|c|}
\hline Enablers & Barriers \\
\hline $\begin{array}{l}\text { Supportive learning environment } \\
\text { Peer support/reduced anxiety }\end{array}$ & $\begin{array}{l}\text { Unsupportive learning environment } \\
\text { Personality differences (anticipated) }\end{array}$ \\
\hline Positive behaviour modeling & Competition \\
\hline Idea sharing & Peer to peer comparison \\
\hline Communication & Different learning styles \\
\hline Competition & Clinical workload \\
\hline Competency availability & Competency availability \\
\hline The clinical education model design & The clinical education model design \\
\hline Student education/preparation & Preceptor education/preparation \\
\hline Planned and unplanned PL & Pre-conceived ideas \\
\hline opportunities & Model evolution, transparency \\
\hline Protected preceptor days & Increased student evaluations \\
\hline Model evolution & \\
\hline
\end{tabular}

conflict management, and the provision of constructive feedback. They reported feeling prepared for working together in a 2:1 model.

Student 6: "We were asked with our partner to come up with 5 rules to live by in our clinical year... and I feel like even though we don't go back and visit those 5 rules every single day we know what's important to our partner and they know what's important to me. One of our rules was to share competencies; so even if we both needed stuff signed off, we'll be fair. It's not that we revisit that chart...but knowing that it's important to my partner, I'm not going to take that away from them."

Preceptor 9: (In response to posters developed by the students during orientation week) "They look very juvenile but they seem to embrace the thing and put a lot of thought into it. They worked together as a team right from the very beginning."

Both students and preceptors praised the five protected preceptor days that were part of the clinical schedule, not present in our previous education models. The protected preceptor days allowed the adoption of workloads that permitted quality-facilitated PL to occur at the bedside, thereby reducing the stress associated with being a preceptor. It was reported that preceptors were able to provide a structured introduction to a clinical area and that students benefited from uninterrupted preceptor attention and being able to provide patient care without time constraints.

Facilitated, unplanned, and PPL opportunities supported by the model were other reported enablers of the 2:1 model. Unplanned PL opportunities started via preceptor facilitation or were independently student initiated and took place at the bedside, within department space, or outside of structured clinical time. For example, in departmental space, students reported opportunities to discuss patient care plans, share knowledge and ideas, and practice their assessment and reporting skills with one another.

Student 5: "You got to practice with each other, it's just like a dress rehearsal. It was helpful because then my partner would say 'Why did they do this?' and you would be like 'I don't even know', and then we would go check that so we'd be better prepared for report."

Comprehensive learning was reported with the PPL activities. Students acknowledged deeper learning when they had to prepare and present the material to their peers. In teaching the material, they had to assure their knowledge and understanding of the material was sufficient prior to their presentation. Group discussions further provided opportunities to expand on knowledge and provide first-hand accounts of their clinical experiences on the presented topic.

\begin{abstract}
Student 9: "It would be the definition of peer learning. We would split into groups, talk about whatever the topic is, create a case study around it, or give a little presentation about it and then educate each other. Then come back as a bigger group and talk about what we've seen in practice...it was interactive, and because you're putting in the extra effort to teach somebody something, you understand things a lot better."
\end{abstract}

It was also reported that there was a natural progression noted in regards to the PL strategies from facilitated PL activities used at beginning of the clinical year to more extensive use of unplanned PL. Preceptors and students noted these to be equally important as they promote the engagement of students in discussion and development of critical thinking skills.

Student 5: "I think peer learning is great at the start especially, but as you're progressing you become more confident in your skills and the global patient picture; you have more confidence similar to that of the staff. I don't think you need so much that cushion to rely on, to ask questions and stuff."

Preceptor 20: "As a preceptor I saw them less towards the end because I think they were able to walk off and discuss stuff by themselves and 
come to a decision as opposed to always coming to me specifically and asking me a question."

Preceptor 2: "They learn just as much inferred knowledge and information with the preceptor talking less by the end."

In addition to the natural progression of PL strategies, the evolution of the model was also articulated as being another important enabler of an effective RT clinical program. There was a notable shift from a 2:1 model used in the beginning of the clinical year, towards a 1:1 model as the clinical year progressed, as directed by the preceptors and students themselves. This evolution was not necessarily time-bound but based upon clinical and critical thinking skill development as well as situational variables.

Student 12: "I felt the progression was very smooth; I can't remember exactly when I went to the one to one. I felt that because I can't remember I think it was at the right time and I didn't feel nervous about it or anything so I think the progression of it was really well done."

\section{Theme 2: Barriers}

The main categories of reported "barriers" included the clinical education model design and an unsupportive learning environment.

\section{The clinical education model design}

Although the new model was introduced to staff at an annual professional development day, an additional education day concentrating on how to facilitate PL strategies was offered to staff on a voluntary basis. Thus, not all preceptors had the opportunity to explore and discuss the principled benefits that a 2:1 model supports. The ambiguity of participants' understanding for implementation of the new clinical model was found to be an important barrier of the model in the RT clinical program.

Preceptor 8: "It seems like you've created something purely out of necessity and now you're trying to make it a lot more than what it is... putting a name to it doesn't make it more valid that what it really is, an over abundance of students and no placements for them."

Preceptor 5: "From what I understand, we moved to this model so that students would benefit from bouncing ideas off of each other, discussions, and learning from each other's mistakes... and if they did something well, then they could learn from that too."

Thus, further education on optimally facilitating a PL approach along with clearer transparency on the evolution of the model to support student autonomy as the year progressed were identified as common concerns. The current literature identifies that preceptors may not feel adequately supported to facilitate PL [5, 16], reporting the need to develop organizational skills, feedback skills, and strategies to cope with the dual role of patient care provider and educator $[2,5,17]$. The preceptors in our study reported varying levels of preparedness, identifying that further education on how to effectively support PL using a 2:1 model should be forefront in subsequent clinical years.

Preceptor 2: "I've come up with my own strategies whereas if there were already successful strategies, maybe disseminating that-opportunities to get together to talk about our experiences and to say...this worked for me, this didn't work for me. Historically we've always just done that with the 1:1, it just evolved over time. But 2 years into this we're young at it I guess."

Student 5: "We hesitated in answering questions so not to talk over each other but then I worried it seemed we didn't know the answer. Instead of doing open ended questions, direct the questions to a student and then be like 'you can ask your partner if you need but I want you to answer this one' more directing questions versus open questions."

Student 19: "Going in I thought we were going to be paired together the entire time. I think it was a good thing that we got divided because I think if we were paired the whole time we wouldn't have been able to complete all of our competencies when we wanted to."

\section{Unsupportive learning environment}

Concerns reported in the interviews on 2:1 models were similar to those reported in the literature for other health care professions. There were concerns regarding the competition for attainment of clinical competencies, peer to peer comparison, increased student evaluations, and anticipated lack of preparation for independent practice.

Student 10: "I think there was competition but mainly around competencies. Because we need to get these done, so of course that is at the back of our minds."

Preceptor 9: "I definitely don't say anything to them like 'Oh you didn't do that as well as so and so.' I would never say that but in my mind I do compare them."

Student 5: "I know my partner has strengths in areas where I'm weaker, and I'm sure the staff can see that and I don't want to be judged for it."

The extent of clinical workload was also seen as a barrier to effectively supporting PL. Following protected preceptor days, preceptors commented on the challenge of balancing clinical workload with high student supervision demands. Difficulty in supervising two students mostly arose midway through the clinical rotation, as students' independence grew but still required a high level of supervision from their preceptors. Despite having an understanding that the preceptor role needs to accommodate the patient workload, preceptors found the delegation of some of their assigned activities to peers challenging. A balance between patient assignments and student supervision may need to be facilitated by RT leadership. Towards the end of the student clinical rotation when they were starting to work independently and required minimal direct supervision, there was noted value in having two students assigned with one preceptor. It was at this stage that the model shifted to a 1:1 model to increase their patient care assignments and support students in demonstrating autonomy.

Preceptor 10: "I need to be monitoring that student very closely to make sure that they are doing the things they are supposed to be doing and if there are two of them there, I can't divide my attention."

Preceptor 2: "During the earlier stages, expect a higher workload dealing with 2 students, but then have the realization that it really only lasts a month or two, and then it starts to lighten as they grow in their confidence and abilities."

Both preceptor and students predicted personality differences to be a possible obstacle to a successful PL program, but this was not experienced with this research group. Although the students commented on the desire to have input into their peer pairing, they did not express concerns with their peer partner. Peer pairs were assigned by the educational institution and were selected based on anticipated personality fit and academia. Suggestions for model improvements included future input by subsequent students in picking their peer partner by selecting three peers they would like to work with, from which the institution would then select from. However, learning the skills to work with different personality types and to practice teamwork based on a professional relationship rather than a pre-existing friendship can only further prepare students for working in the real world.

\section{DISCUSSION}

By increasing the ratio of students to clinical preceptors, a 2:1 model increases the capacity for more student clinical placements; a pragmatic solution for clinical sites looking to do just this in the face of higher 
student placement demands. However, if the benefits of a 2:1 model are only based on this pragmatic drive, then its full potential is not understood [8]. The educational benefits of a 2:1 model have its foundation in $\mathrm{PL}$, but an effective PL approach involves more than just co-locating students in the clinical environment.

With a PL approach there is less expectation on preceptors to provide the traditional transfer of knowledge directly to students, and students are instead encouraged to take on more responsibility for their learning. The preceptor must cultivate the students' critical thinking by asking questions that challenge the students to evaluate and build on the current or hypothetical situation [18]. Thus, optimizing education for preceptors on how to support discussions and foster dialogue that enhances student critical thinking will be key in further strengthening the RT PL approach at UHN. Additionally, an understanding of differing definitions of clinical educational success amid preceptors and students, clarity on the PL methods that support the model, and further education on the defined roles and responsibilities of all parties needs to be disseminated.

The most prominent finding emerging from the interviews illustrated that a 2:1 model strongly contributes to a supportive learning environment where decreased anxiety and peer encouragement increases the exploring of ideas through an "ask-anything culture". The non-hierarchal structure of a 2:1 model may encourage students to be more open about misconceptions or ask questions about new knowledge and skills [19]. Although the majority of study participants reported that a 2:1 model supports reduced anxiety for students during their clinical training, only a handful of participants linked this reduced anxiety as supporting their learning. In a learning context, anxiety can affect the ability of receiving information, its processing, and retrieving it when necessary [20]. There have been many stressors reported in the literature experienced by students associated with the clinical setting. These have included feelings of ill preparation for practice, fear of making mistakes, issues related to death and dying, and problematic interprofessional relationships [21, 22]. Thus, the potential impact that peer support can have on students' cognitive learning to help their success and improve patient safety may be under-recognized by both staff and students.

From an organizational perspective, participants acknowledged that a key factor supporting the success of this new education model is a direct result of the protected preceptor days included in the clinical schedule. As RT work is often dictated by urgent care needs, time constraints, and involves the constant reprioritization of workload, protected preceptors days provide uninterrupted time for preceptors to facilitate learning at the bedside. In having protected preceptor days at the start of each new clinical area, preceptors were able to educate students on the UHN RT roles, responsibilities, equipment, and daily routines-in addition to supporting facilitated PL. Participants felt this was an important step before pairing students with a staff juggling the role of preceptor while carrying a pager and patient caseload. As a department we were able to argue that the increased departmental cost required to support the additional staffing complement needed for the five protected preceptors days for each student pair is offset by the increased student numbers that a 2:1 model supports, resulting in a larger hiring pool of student graduates. As there is a significant increase in cost associated with the orientation of external RT hires, a 2:1 model provides a greater number of potential new staff to select from to support growing program demands.

Despite the intention for students to be paired with preceptors in a 2:1 model for the entire clinical year, a transition to a 1:1 model organically emerged as the clinical year progressed. This change was both student and staff driven to further support the transition of students from interdependence to independence. Perhaps unique to our clinical program at UHN was the capability for this evolution as our staffing complement can support a single student being paired with a single preceptor while concurrently scheduled in the same clinical area as their peer pair. This helps alleviate the amount of student evaluations required by a single preceptor, while still supporting unplanned PL towards the end of the clinical year.
As the department becomes more familiar with how the 2:1 model and PL strategies best support our clinical education program, further transparency can be communicated to future UHN RT students and staff. Leadership may need to support the restructuring of day-to-day clinical coverage to avoid the need for preceptors to work harder, but instead to work differently. Because RTs can provide coverage to a large number of patients-and may even provide coverage across different areas within an organization-the nature of RT clinical work is unpredictable, making a predictable structured 2:1 model unrealistic. Our educational program will likely continue to evolve, dependant on available resources and the clinical environment. A 2:1 model can set the foundation for a PL educational program but the structure can be customized to health care professions' individual needs.

\section{LIMITATIONS}

This study was conducted with RT students from one school of respiratory therapy and included a large acute care setting offering many opportunities for competency skillsets in a resource-rich institution. This may have contributed to the success of the 2:1 model in our clinical setting. We acknowledge that results were interpreted on a small sample, as well as the nature of self-reporting on variables, and are therefore not generalizable to every RT clinical program. Because of feasibility issues, we did not investigate if barriers were truly reflective of the 2:1 model structure itself, or were generalizable for any type of RT-run clinical education model. While questions of model evaluation are continuing to be addressed as part of the implementation process, these evaluative questions are out of scope for this particular paper. The intention of this study was to describe the model and to explore participant perceptions, specifically their perceptions of PL.

\section{CONCLUSION}

A PL approach using a 2:1 model in the UHN RT clinical program was shown to support an increased need for student capacity and was found to be a satisfying clinical education model for both students and preceptors. Successful implementation of a 2:1 model in the unique environment of an RT clinical setting not only requires adequate preceptor and student education, but the application of different types of PL opportunities, protected preceptor time, and flexibility in reorganizing clinical workload.

Study participants felt that the improved critical thinking and student engagement opportunities that the 2:1 model offers has the potential to enhance student learning and overall improves the clinical education experience within our high reliability organization. Despite no longer being faced with a placement capacity issue of RT students at UHN for this clinical student year, a 2:1 model will continue to be forefront in our clinical education program.

\section{REFERENCES}

1. Smith P, Corso L, Cobb N. The perennail struggle to find clinical placement opportunities: A Canadian national survey. Nurse Educ Today 2010;30:798-803. doi: 10.1016/j.nedt.2010.02.004

2. Dawes J, Lambert P. Practice educators' experiences of supervising two students on allied health practice-based placements. J Allied Health 2010;39(1):20-7.

3. Aston L, Molassiotis A. Supervising and supporting student nurses in clinical placements: The peer support initiative. Nurse Educ Today 2003;23(3):202-10. doi: 10.1016/S0260-6917(02)00215-0.

4. Baldry Currens, J. The 2:1 Clinical Placement Model - Review. Physiotherapy 2003;89(9):540-54. doi: 10.1016/S0031-9406(05)60180-0.

5. Baldry Currens J, Bithell CP. The 2:1 Clinical placement model. Perceptions of clinical educators and students. Physiotherapy 2003;89(4): 204-18. doi: 10.1016/S0260-6917(02)00215-0.

6. Stenberg M, Carlson E. Swedish student nurses' perception of peer learning as an educational model during clinical practice in a hospital setting-an evaluation study. BMC Nurs 2015;14(48). doi: 10.1186/ s12912-015-0098-2.

7. Sevenhuysen S, Nickson W, Farlie M, et al. The development of a peer assisted learning model for the clinical education of physiotherapy students. J Peer Learn 2013;6(4):30-45 
8. Boud D, Cohen R, Sampson J. Peer learning in higher education: Learning from and with each other. London: Kogan Page Limited; 2001.

9. Secomb J. A systematic review of peer teaching and learning in clinical education. J Clin Nurs 2008;17(6):703-16. doi: 10.1111/j.1365-2702. 2007.01954.x.

10. Owens L, Walden D. Peer instruction in the learning labouratory: A strategy to decrease student anxiety. J Nurs Educ 2001;40(8):375-7.

11. Loke A, Chow F. Learning partnership - the experience of peer tutoring among nursing students: A qualitative study. Int J Nurs Stud 2007;44(2):237-44. doi: 10.1016/j.ijnurstu.2005.11.028.

12. Henning J, Tg W, Jones J. Peer-assisted learning in the athletic training clinical setting. J Althletic Training 2006;41(1):102-8.

13. Lincoln M, McAllister L. Peer learning in clinical education. Med Teacher 1993;15(1):17-25. doi: 10.3109/01421599309029007.

14. CRTO: Students: Approved RT Programs. (2017, 07 22). from College of Respiratory Therapists of Ontario. Available at: http://www.crto.on.ca/ student/registration/accredited-schools (Accessed July 22, 2017).

15. Hallet C. Understanding the phenomenological approach to research. Nurse Res 1995;3(2):55-65. doi: 10.7748/nr.3.2.55.s6.
16. Henning J, Weidner T, Snyder M, Dudley W. Perceived frequency of peer-assisted learning in the laboratory and collegiate clinical setting. J Athletic Train 2012;47(2):212-20. doi: 10.4085/1062-6050-47.2.212.

17. O'connor A, Cahill M, McKay E. Revisiting $1: 1$ and 2:1 clinical placement models: Student and clinical educator perspectives. Aust Occupation Therap J 2012;59(4):276-83. doi: 10.1111/j.1440-1630.2012. 01025.x.

18. Gavett E, Peapers R. Critical thinking: The role of questions. Perspect Issues High Educ 2007;10:3-5. doi: 10.1044/ihe10.1.3.

19. Christiansen A, Bell A. Peer learning partnerships: Exploring the experience of pre-registration nursing students. J Clin Nurs 2010;19(5-6): 803-10. doi: 10.1044/ihe10.1.3.

20. Bigdeli S. Affective learning: The anxiety construct in adult learners. Procedia-Social Behav Sci 2010;9:674-8. doi: 10.1016/j.sbspro.2010.12.216.

21. Moscaritolo L. Interventional strategies to decrease nursing student anxiety in the clinical learning environment. J Nurs Educ 2009;48(1):17-23. doi: 10.3928/01484834-20090101-08.

22. Chernomas W, Shapiro C. Stress, depression and anxiety among undergraduate nursing students. Int J Nurs Educ Scholarship 2013;10(1): 255-66. doi: 10.1515/ijnes-2012-0032. 